\title{
Optimal Selection of Cyclic-prefix and Sub-carrier for OFDM Signal in Mobile Satellite Communications Channel
}

\author{
Bin Li, Wei Zheng, Shubo Ren, Jianjun Wu \\ Institution of Advanced Communications, EECS, Peking University, Beijing, 100871 \\ E-mail: sueno@pku.edu.cn
}

\begin{abstract}
Adapted parameters of OFDM signals can bring performance improvement in mobile satellite communications. This paper firstly analyzes the effect of cyclic-prefix (CP) length and sub-carrier bandwidth on system interference and capacity. This effect can be described by a formula which consists of system capacity, CP length and sub-carrier bandwidth. Then using this formula, a method to select the optimal parameters is given. In the end, a numerical calculation is performed to figure out the optimal parameters, considering the multipath delay spread and Doppler spread in satellite channel. It comes up that in satellite channel the $C P$ is not very effective to improve the system performance and a wider sub-carrier bandwidth is desired.
\end{abstract}

Keywords-mobile satellite communication; OFDM; CP length; sub-carrier bandwidth

\section{INTRODUCTION}

Orthogonal Frequency Division Multiplexing (OFDM) technology has advantage of high bandwidth efficiency, flexible resource allocation and multipath mitigation, so it is widely used in modern terrestrial communications. When designing an OFDM based system, several factors such as sub-carrier bandwidth, guard interval, cyclic-prefix, symbol duration and modulation on every sub-carrier should be considered in order to enhance system performance. However, these factors are partly contradictive. For example, more sub-carriers can decrease the spacing of each subcarrier so that the system can better tolerate delay spread, but the opposite is worse performance against Doppler spread and phase noise. For this reason, how to select adaptive parameters for OFDM based systems is a tradeoff problem that should be optimized, and it's also the problem should be considered first when designing an OFDM based system.

Extensive research has been conducted on selection of OFDM parameters. Ref. 2 analyzes how the OFDM parameters affect system interference in frequency-selected fading and time-selected fading channel. The conclusion is that more attention should be paid to the CP length in frequency-selected fading channel, and that shorter CP may lead to larger Inter-Symbol Interference (ISI). When in timeselected fading channel, the conclusion is that more attention should be paid to sub-carrier bandwidth, and that smaller sub-carrier bandwidth will bring larger Inter-Carrier Interference (ICI). Furthermore, in urban environment, multipath effect is strong and causes more ISI than ICI. So in terrestrial urban communications, the $\mathrm{CP}$ length must be larger than the time delay spread in order to minimize the ISI.
Ref. 3 discusses the ICI caused by Doppler spread. Ref. 4 gives a formula of the sub-carrier number and system throughput in the case that CP length is larger than time delay spread, and figures out the optimal sub-carrier number in AWGN channel. Ref. 5 deduces the expression of ICI in under water channel, and proposes a method to dynamically select sub-carrier bandwidth.

With satellite mobile communications are widely used in every field, satellite mobile communications are required to transmit more data in a shorter time, so using OFDM technology in satellite mobile communications becomes a hot research topic. Nevertheless, some conclusion in terrestrial communications can't be simply brought into satellite scenario, because the characteristics of satellite channel are much different from those of terrestrial channel. For instance, satellite channel has smaller time delay spread, bigger elevation angle and larger Doppler shift caused by the movement of satellite. All these differences make the optimal parameters of terrestrial OFDM based systems not applicable to satellite mobile systems. This paper aims to analyze how CP length and sub-carrier bandwidth influence system capacity, and considers shortening CP length so as to improve system capacity according to the fact that satellite channel has weaker time delay spread. Then every possible $\mathrm{CP}$ length and sub-carrier bandwidth is enumerated to calculate the system capacity, and reasonable parameters which result in higher capacity is selected as the optimal parameters in satellite mobile communications.

The rest of the paper is organized as follows. Section II describes the OFDM based system model. A simplified model without modulation in each sub-carrier is used in this paper. Section III gives the expression of ICI and ISI. By using these expressions, Signal to Interference Ratio (SIR) and system capacity can be calculated. Then a method to optimize the CP length and sub-carrier bandwidth for OFDM is presented. This method is used to figure out the optimal CP length and sub-carrier bandwidth for satellite channel in Section IV. Section V gives the conclusion of this paper.

\section{SYSTEM MODEL}

A simplified model of modulated data of OFDM system transmitting in the satellite channel is shown in Fig. 1, where IFFT is short for Inverse Fast Fourier Transform and FFT is the abbreviation of Fast Fourier Transform. 


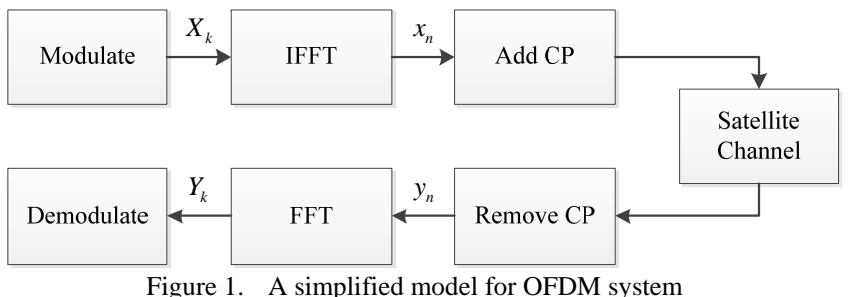

$X_{k}$ represents the modulated complex signal, as shown in Fig. 1. The discrete expression of the transmission signal after IFFT is

$$
X_{n}=\sum_{k=0}^{N-1} X_{k} e^{j 2 \pi f_{k}\left(n T_{S}\right)} \quad 0 \leq n \leq N-1
$$

where: $N$ is the number of points in IFFT, which equals the sub-carrier number when the system is full loaded; $f_{k}$ is the frequency of the $k$ th sub-carrier bandwidth; $T_{S}$ is the symbol duration, which is equal to the reciprocal of the sub-carrier spacing. CP needs to be added to each OFDM symbol to mitigate multipath effect. Assuming the CP length is $G$, the transmission signal is given by

$$
X_{n}=\sum_{k=0}^{N-1} X_{k} e^{j 2 \pi f_{k}\left(n T_{S}\right)} \quad-G \leq n \leq N-1
$$

As shown in Fig. 1, $y_{n}$ stands for the signal passed through the multipath channel $h=\left[h_{0}, h_{1}, \ldots, h_{M-1}\right]$, sampled every $T_{S} / N$, and with CP removed afterwards. When $M \leq G$, the CP length of OFDM symbol overlaps the maximum channel delay spread, so that the orthogonally between symbols is not destroyed and that no ISI exists. However, when $M>G$, the OFDM symbol received is affected by the last transmission signal, as shown below

$$
y_{n}= \begin{cases}\sum_{m=0}^{M-1} h_{m} x_{n-m} & M-G \leq n \leq N-1 \\ \sum_{m=0}^{G+n} h_{m} x_{n-m}+\sum_{m=G+n+1}^{M-1} h_{m} x^{\prime} & 0 \leq n \leq M-G-1\end{cases}
$$

where $X^{\prime}$ is the time domain signal of the last OFDM signal, which can be seen as a noise signal with the same power average as $x_{n}$. In the case of $0 \leq n \leq M-G-1$, the second term brings in ISI.

After receiving the signal, the receiving end needs to perform FFT to obtain the received data. In case $M \leq G$, the expression of received signal derived by Ref. 4 is

$$
Y_{k}=\sum_{n=0}^{N-1} X_{k} H_{k}(n)+\sum_{n=0}^{N-1} \sum_{p=0, p \neq k}^{N-1} X_{p} H_{p}(n) e^{j 2 \pi\left(f_{p}-f_{k}\right) n T_{s}}
$$

where the second term is ICI, and $H_{k}(n)$ is the channel response in frequency domain with the consideration of Doppler spread,

$$
H_{k}(n)=\sum_{m=0}^{M-1} h_{m} e^{-j 2 \pi\left[f_{k} n T_{S}+\left(f_{k}+f_{k m}\right)(n-m) T_{S}\right]}
$$

\section{METHOD OF SELECTING OFDM PARAMETERS}

In order to calculate the system SIR, specific expression of ISI and ICI should be taken into consideration. ISI of the $k$ th sub-carrier can be obtained by processing the second term of Eq. (3) in the case of $0 \leq n \leq M-G-1$ by FFT, which is calculated as

$$
I S I_{k}=\sum_{n=0}^{M-G-1}\left(\sum_{m=G+n+1}^{M-1} h_{m} x^{\prime}\right) e^{-j 2 \pi f_{k} n T_{S}}
$$

When the Doppler frequency of the channel of the $k$ th sub-carrier is $f_{k m}$, the second term of Eq. (4) is ICI, if not considering the influence of ISI. But taking the existence of ISI into account, these two terms affect each other. A simplification is made in this paper that the overlap of ISI and ICI is classified as ISI, as expressed by Eq. (6), and that only the ICI between wanted signals is calculated. Referred to the classified discussion of Eq. (3), the ICI expression of the $k$ th sub-carrier in Ref. 4 can be modified as

$$
\begin{aligned}
I C I_{k} & =\sum_{n=M-G}^{N-1} \sum_{p=0, p \neq k}^{N-1} X_{p} H_{p}(n) e^{j 2 \pi\left(f_{p}-f_{k}\right) n T_{S}} \\
& +\sum_{n=0}^{M-G-1} \sum_{p=0, p \neq k}^{N-1} X_{p} H_{p}^{\prime}(n) e^{j 2 \pi\left(f_{p}-f_{k}\right) n T_{S}}
\end{aligned}
$$

where $H^{\prime}{ }_{p}(n)$ is the channel response in frequency domain for the signal with ISI removed,

$$
H_{p}^{\prime}(n)=\sum_{m=0}^{G+n} h_{m} e^{-j 2 \pi\left[f_{p} n T_{S}+\left(f_{p}+f_{p m}\right)(n-m) T_{S}\right]}
$$

Without loss of generality, channel noise and co-channel inter-cell interference are not considered, and the SIR of the $k$ th sub-carrier is given by 


$$
\begin{aligned}
& S I R_{k}=\frac{\left\langle\operatorname{signal}_{k}^{2}\right\rangle}{\left\langle I S I_{k}^{2}\right\rangle+<{\left.I C I_{k}^{2}\right\rangle}^{2}} \\
& =\frac{\left.\left.\left\langle Y_{k}^{2}\right\rangle-<I S I_{k}^{2}\right\rangle-<I C I_{k}^{2}\right\rangle}{\left.\left.<I S I_{k}^{2}\right\rangle+<I C I_{k}^{2}\right\rangle}
\end{aligned}
$$

The modulation and coding scheme is of no concern, because it can be controlled by Adaptive Modulation and Coding (AMC) to guarantee the optimal throughput. Using Shannon formula, the upper limit of each sub-carrier is calculated as

$$
C_{k}=\Delta f \log _{2}\left(1+S I R_{k}\right)
$$

where $\Delta f$ is the sub-carrier bandwidth. Assuming each subcarrier is loaded with data, the theoretical capacity of the system is

$$
C_{\max }=\beta\left\lfloor\frac{B_{C}}{\Delta f}\right\rfloor \Delta f \sum_{k=0}^{\left\lfloor\frac{B_{c}}{\Delta f}\right\rfloor-1} \log _{2}\left(1+S I R_{k}\right)
$$

where $B_{C}$ is the total bandwidth of the system, $\beta=T_{S} /\left(T_{S}+T_{C P}\right)$ is the bandwidth efficiency of the system, and $\lfloor\bullet\rfloor$ means to round down to the nearest integer.

The relationship of CP length, sub-carrier bandwidth and system theoretical capacity can be obtained by Eq. (11). In the same conditions, it is reasonable to think that the OFDM parameters which maximize the system theoretical capacity are the optimal results. The specific process is as follows.

a) Determine the bandwidth and sub-carrier frequency of the system, and a specific channel response $\mathrm{h}$ referred to the satellite channel model given by Ref. 8;

b) Provide the Doppler spread value for the satellite channel in condition of a motion speed of $30 \mathrm{~km} / \mathrm{h}, 120 \mathrm{~km} / \mathrm{h}$ and $350 \mathrm{~km} / \mathrm{h}$. Both GEO satellite and LEO satellite need to be considered, due to their optimal parameters may be different;

c) Enumerate different values of CP length and subcarrier bandwidth, and calculate the SIR of each sub-carrier by substituting the channel parameters got from above into Eq. (6)-(9). The SIR of each sub-carrier is different because of different frequency of each such-carrier;

d) Use Eq. (11) to obtain the theoretical capacity of the system. Repeat (a) (c) for numerous times to get the average value by reason of the randomness of the channel. At last, for different values of $\mathrm{CP}$ length, plot a curve diagram of the system capacity to select the optimal OFDM parameters, with the horizontal axis showing the sub-carrier bandwidth.

\section{NUMERICAL RESULTS}

In this paper, the setting of OFDM parameters is calculated based on the satellite broadband channel model.
Adopting channel model $\mathrm{C}$, the specific tap delay value and amplitude are shown in Table. 1.

TABLE I. TABLE TyPe StYles

\begin{tabular}{ccccc}
\hline $\begin{array}{c}\text { Tap } \\
\text { No. }\end{array}$ & $\begin{array}{c}\text { Tap delay } \\
\text { value (ns) }\end{array}$ & $\begin{array}{c}\text { Amplitude } \\
\text { distribution }\end{array}$ & $\begin{array}{c}\text { Average } \\
\text { amplitude }\end{array}$ & $\begin{array}{c}\text { Rice } \\
\text { factor } \\
(\mathrm{dB})\end{array}$ \\
\hline 1 & 0 & $\begin{array}{c}\text { Rice } \\
\text { Rayleigh }\end{array}$ & 0.0 & 3 \\
2 & 60 & Rayleigh & -17.0 & - \\
3 & 100 & Rayleigh & -19.3 & - \\
4 & 130 & Rayleigh & -22.1 & - \\
5 & 250 & & - \\
\hline
\end{tabular}

LEO and GEO satellite system are both taken into consideration. And in LEO satellite system, Doppler shift caused by the movement of satellite can be estimated and eliminable, so the channel Doppler spread is mainly generated by the movement of terminal. With the same carrier frequency, the Doppler spread of LEO satellite system and that of GEO satellite system are the same. Ref. 7 mentions that in condition of a motion speed of $350 \mathrm{~km} / \mathrm{h}$, the ratio of the maximum Doppler spread and the carrier frequency is 3e-7, and that the maximum Doppler spread can reach $600 \mathrm{~Hz}$ when the carrier frequency is $2 \mathrm{GHz}$.

It can be seen from Table. 1 that the maximum delay value of the multipath channel is 250ns. The multipath delay value is slightly larger than that, when the sampling interval is smaller the multipath delay. The CP length is set to 0ns, $100 \mathrm{~ns}, 250 \mathrm{~ns}$ and $400 \mathrm{~ns}$ to calculate the system capacity, the carrier frequency is set to $2 \mathrm{GHz}$, and the system bandwidth is set to $5 \mathrm{MHz}$. When the sub-carrier bandwidth varies from $15 \mathrm{kHz}$ to $1500 \mathrm{kHz}$, the system capacity calculated using Eq. (11) is shown in Fig. 2.

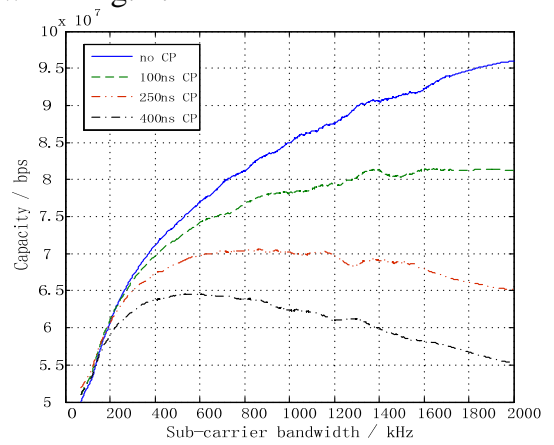

Figure 2. Influence of sub-carrier bandwidth on system capacity with different values of CP length

In terrestrial OFDM communication systems (such as LTE), CP length is set to 4us 6us, which is about ten times as the delay spread of the satellite channel. Oversize value of CP length brings down the system bandwidth efficiency, leading to decrease of system performance. Therefore, in the satellite channel system with a smaller delay spread, the value of CP length in terrestrial systems is optimizable. It can be seen from Fig. 2 that when CP length is 400ns, slightly larger than the channel delay spread, the system capacity does not fall down until sub-carrier bandwidth reaches about $500 \mathrm{kHz}$. While $\mathrm{CP}$ length is less than the channel delay spread, system capacity is more influenced by the bandwidth efficiency, because the power of each path of the multipath channel is very small. In this case, shorter CP 
length leads to more system performance improvement, when sub-carrier bandwidth is larger than $200 \mathrm{kHz}$.

As a result, given the characteristics of satellite channel such as small multipath delay spread, low multipath power and bigger Doppler spread, CP length which is less than delay spread and bigger sub-carrier bandwidth should be set, in order to make the system bandwidth efficiency not decline fast because of sub-carrier bandwidth getting larger. It can be seen from Fig. 2 that, when sub-carrier bandwidth is larger than $200 \mathrm{kHz}, \mathrm{CP}$ can be removed to guarantee the maximum of bandwidth efficiency. In consideration of the flexibility of system resource allocation, the number of FFT points can be set to 16 , meaning the sub-carrier bandwidth is $312.5 \mathrm{kHz}$. The sub-carrier bandwidth of an actual system can be slightly less than that because of the existence of guard bandwidth.

\section{CONCLUSION}

In OFDM based satellite mobile communications, time delay spread of satellite channel is much smaller than that of terrestrial channel, so the CP length can be shortened in order to improve the system performance. This paper uses the ISI and ICI expression to show how CP length and subcarrier bandwidth influence system capacity. Then via numerical calculation, the interference and capacity of OFDM based system in satellite channel is figured out. The channel used in this paper is in S band and the system bandwidth is $5 \mathrm{MHz}$. The numerical result shows that in satellite channel, ISI caused by time delay spread is extremely weak, and most of interference is composed of ICI which is caused by Doppler spread. The result also shows that setting a large sub-carrier bandwidth and removing CP can restrain the interference. Finally considering the requirement of resource allocation, this paper sets no CP and $312.5 \mathrm{kHz}$ sub-carrier bandwidth for the OFDM based satellite mobile communication system described here. And for other OFDM based systems, according to its frequency band, system bandwidth, channel time delay spread, Doppler spread and other characteristics, the optimal CP length and sub-carrier bandwidth can also be selected with this method.

\section{ACKNOWLEDGMENT}

This work is supported by the National Science Foundation of China (Grant No. NFSC \#61071083) and the National High-Tech Research and Development Program of China (863 Program), No. 2012AA01A506.

Corresponding author: Jianjun Wu; phone: +86-1062752848; e-mail: just@pku.edu.cn.

\section{REFERENCES}

[1] R. Van Nee, and R. Prasad, "OFDM for Wireless Multimedia Communications 1st,” Artech House Publishers, 2000, Chaps. 3

[2] H. Steendam, and M. Moeneclaey, "Analysis and Optimization of the Performance of OFDM on Frequency-selected Time-selective Fading Channels,” IEEE Trans. On Comm., Vol. 47, No. 12, December 1999

[3] P. Robertson, and S. Kaiser, "The Effects of Doppler Spreads in OFDM(A) Mobile Radio Systems,” Proc. IEEE VTC 50th, Vol. 1, pp. 19-20, September 1999

[4] S. S. Das, E. De Carvalho, and R. Prasad, "Performance Analysis of OFDM Systems with Adaptive Sub Carrier Bandwidth," IEEE Trans. On Wireless Comm., Vol. 7, No. 4, pp. 1117-1122, April 2008

[5] S. Ahmed, and H. Arslan, "Evaluation of Frequency Offset and Doppler Effect in Terrestrial RF and in Underwater Acoustic OFDM Systems,” Proc. IEEE MILCOM, 2008

[6] I. Ali, N. Al-Dhahir, and J. E. Hershey, "Doppler Characterization for LEO Satellites,” IEEE Trans. On Comm., Vol. 46, No. 3, pp. 309-311, March 1998

[7] B. Li, X. Luan, Y. Si, S. Ren, and J. Wu, "Selection of Sub-carrier Bandwidth in OFDM based Mobile Satellite Communication," IEEE ICIST, 2012

[8] ITU-R. M. 1225, Guidelines for Evaluation of Radio Transmission Technologies for IMT-2000, ITU, pp. 38-39, 1997 